\title{
ADHESION BETWEEN A POWER-LAW INDENTER AND A THIN LAYER COATED ON A RIGID SUBSTRATE
}

\author{
UDC 539.6
}

\begin{abstract}
Antonio Papangelo ${ }^{1,2}$
${ }^{1}$ Politecnico di Bari, Department of Mechanics, Mathematics and Management, Italy ${ }^{2}$ Hamburg University of Technology, Department of Mechanical Engineering, Germany

Abstract. In the present paper we investigate indentation of a power-law axisymmetric rigid probe in adhesive contact with a "thin layer" laying on a rigid foundation for both frictionless unbounded and bounded compressible cases. The investigation relies on the "thin layer" assumption proposed by Johnson, i.e. the layer thickness being much smaller than the radius of the contact area, and it makes use of the previous solutions proposed by Jaffar and Barber for the adhesiveless case. We give analytical predictions of the loading curves and provide indentation, load and contact radius at the pull-off. It is shown that the adhesive behavior is strongly affected by the indenter shape; nevertheless below a critical thickness of the layer (typically below $1 \mu \mathrm{m}$ ) the theoretical strength of the material is reached. This is in contrast with the Hertzian case, which has been shown to be insensitive to the layer thickness. Two cases are investigated, namely, the case of a free layer and the case of a compressible confined layer, the latter being more "efficient", as, due to Poisson effects, the same detachment force is reached with a smaller contact area. It is suggested that high sensitive micro-/nanoindentation tests may be performed using probes with different power law profiles for characterization of adhesive and elastic properties of micro-/nanolayers.
\end{abstract}

Key Words: Adhesion, Layer, JKR model, Adhesion Enhancement

\section{INTRODUCTION}

Adhesion is a much debated topic in contact mechanics covering different fields of application, from adhesion of rough surfaces [1-4] to bioinspired adhesive mechanisms [5, 6]. Nature has inspired different researchers to try to reproduce the same design strategy adopted by insects such as the "famous" gecko, or to develop an "optimal" profile to reach theoretical adhesive strength on a substrate [6-8]. The progress of technology allows us today to "design"

Received January 02, 2018 / Accepted February 02, 2018

Corresponding author: Antonio Papangelo

Department of Mechanics, Mathematics and Management, Politecnico di Bari, Viale Japigia 182, 70126 Bari, Italy E-mail: antonio.papangelo@poliba.it 
surface topography down to the nanoscale. Nanopatterned surfaces, with repeating pillars [9] or dimples [10], are nowadays inspiring many researchers to develop pressure sensitive adhesive mechanisms [10-12]. The majority of scientific literature has focused on the case of halfspace geometry; nevertheless, the development of microelectromechanical systems (MEMS), anti-wear coatings, microelectronics, pressure sensitive adhesive, multilayer coatings calls for a detailed understanding of the contact behavior of the layered surfaces in presence of adhesion. Some authors have dealt with axisymmetric contact of an elastic layer supported by a rigid foundation in adhesiveless and adhesive cases, both analytically $[13,14]$ and numerically [15]. It has been shown that for the Hertzian profile the pull-off force does not depend on the elastic properties of the material, similarly to the classical solution of Johnson-Kendall-Roberts (JKR) valid for halfspace geometry $[16,17]$. Argatov et al. [18] also studied the indentation of an elliptic paraboloid profile in contact with a transversely isotropic layer supported by a rigid foundation in the compressible and incompressible case. To unveil the effect of the indenter profile, in this paper we study the adhesive indentation of an axisymmetric frictionless rigid punch with a power-law profile, which indents a compressible "thin layer" on a rigid foundation in both the bounded and unbounded case. The "thin layer" approximation was first proposed by Johnson [19], who assumed that layer thickness $b$ is much smaller than radius of contact $a$, i.e. $b<<a$, so that the plane sections remain plane upon deformation. The same hypothesis was used by Jaffar [20], who expanded the analysis to the axisymmetric case and later by Barber [21] who generalized the formulation for an arbitrary three dimensional problem. Following Barber [21] we will derive the adhesive solution in the case of short range adhesion (the so-called JKR limit). To this end the energetic derivation of the original JKR model will not be repeated anew. It is in fact known that the adhesive JKR solution can be directly obtained from the adhesiveless solution [22] (see also [23-25]), i.e. indentation $\delta$ is

$$
\delta=\delta_{1}-\sqrt{2 w A^{\prime} / P_{1}^{\prime \prime}}
$$

where $\delta_{1}$ is the adhesiveless indentation, $w$ is the work of adhesion, $A^{\prime}$ is the first derivative of contact area and $P_{1}$ " the second derivative of the adhesiveless load with respect to $\delta_{1}$. Then, adhesive load $P$ is

$$
P=P_{1}-P_{1}^{\prime} \sqrt{2 w A^{\prime} / P_{1}^{\prime \prime}}
$$

where $P_{1}^{\prime}$ is the first derivative of the adhesiveless load with respect to indentation $\delta_{1}$. Notice that $(1,2)$ are approximated for a general three-dimensional contact, but they are exact for an axisymmetric or line contact; hence we are not adding any other approximation except the "thin layer" assumption. Recently a further generalization of the approach suggested by Argatov [22] has been proposed [26], which, in boundary element simulations, introduces a mesh dependent criterion (based on the balance between elastic energy release and adhesion work) to numerically solve adhesive contacts of complex shapes, which in principle can be easily generalized to the case of layered systems.

In this paper we will extend the asymptotic solutions for adhesiveless thin layer problems found by Johnson [19], Jaffar [20], Barber [21] to the JKR adhesive case. We shall then discuss implications, particularly regarding the dependence of pull-off and loading curves on the geometry of the profile suggesting the strategies to obtain "optimal" adhesion. The analytical results obtained may be of interest for elastic and adhesive property characterization of thin films, through nano/microindentation test, particularly when a polymeric coating is adopted over metals. 


\section{METHODS}

We study indentation by a rigid frictionless punch on a thin layer on a rigid substrate in presence of short range adhesion (JKR type [16]). To this end the adhesiveless relations load-indentation and area-indentation are needed. We follow the line of argument of Barber [21], who provided the solution of the adhesiveless problem, for a general three dimensional profile of the punch, for the case of both a frictionless unbounded layer and a bounded compressible layer. The Cartesian coordinate system is set as follows: the contact surface of the layer coincides with plane $z=0$ while two-dimensional spatial coordinates $x_{1}$, $x_{2}$ lie in the plane of the layer. We restrict our analysis to the case where layer thickness $b$ is much smaller than contact radius $a$, i.e., $b<<a$, so that the deformation through the layer is homogeneous; thus, the plane section remains plane upon compression. This corresponds to the original Johnson's approximation, which implies that the in-plane displacements of the layer with components $u_{1}, u_{2}$ are independent of $z$. For the case of frictionless foundation Barber [21] showed that the contact pressure is

$$
p\left(x_{1}, x_{2}\right)=\frac{E^{*}}{b} \xi\left(x_{1}, x_{2}\right)
$$

where indentation $\xi$ is the local interpenetration between the indenter and the layer if it did not deform, $E^{*}$ is the effective elastic modulus $E^{*}=E_{1} /\left(1-v^{2}\right), v$ the Poisson's ratio, $b$ the layer thickness. For the case of bounded compressible layer Barber [21] obtained

$$
p\left(x_{1}, x_{2}\right)=\frac{E^{*} Z}{b} \xi\left(x_{1}, x_{2}\right)
$$

where $Z=(1-v)^{2} /(1-2 v)$. The general three dimensional adhesiveless solution could be transformed into the corresponding JKR adhesive solution with the approximation that the "contact shape" is the same in the adhesive and adhesiveless solutions [22-25]. However, here we are dealing with axisymmetric contact; therefore, no approximations are introduced [22-25]; thus, the solution will be exact, provided that the original Johnson's approximation of a "thin layer" is fulfilled.

\section{AXISYMmetric CONTACT WITH POWER LAW ProfiLe}

\subsection{Frictionless unbounded layer}

Consider the indentation by a rigid axisymmetric frictionless indenter with power law profile $r^{k} /\left(k R^{k-1}\right)$ on a thin layer supported by a rigid foundation ( $r$ is the radial coordinate and $R$ a reference length, see Fig. 1).

For the case of a frictionless unbounded layer the contact pressure is given by Eq. (3), and hence

$$
p(r)=\frac{E^{*}}{b}\left(\delta_{1}-\frac{r^{k}}{k R^{k-1}}\right)
$$

where $\delta_{1}$ is the adhesiveless indentation. 


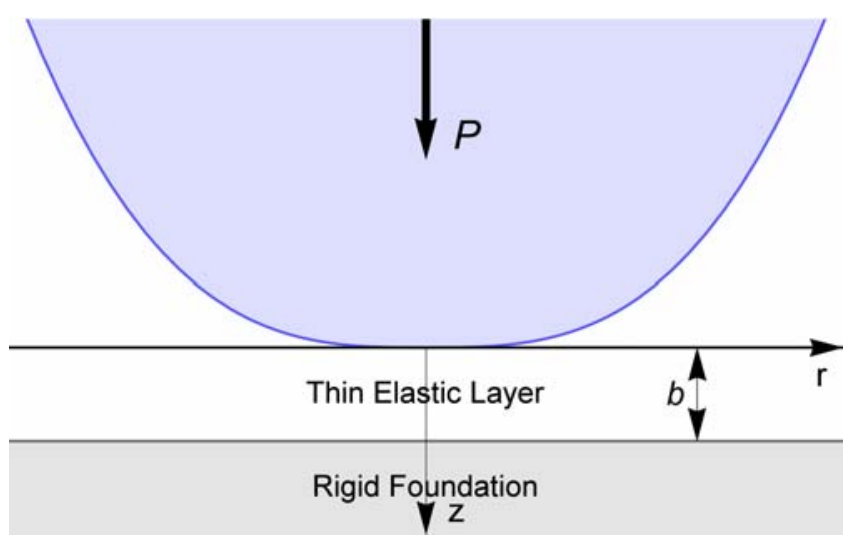

Fig. 1 Schematic representation of an axisymmetric power law profile in contact with a thin elastic layer of thickness $b$ supported by a rigid foundation

The total load reads

$$
P_{1}=\frac{\pi}{k+2} \frac{E^{*}}{b} \frac{a^{k+2}}{R^{k-1}}
$$

where $a$ is the contact radius and the subscript "1" stands for the adhesiveless solution. In fact from $p(a)=0$, the adhesiveless indentation $\delta_{1}$ is

$$
\delta_{1}=\frac{1}{k} \frac{a^{k}}{R^{k-1}}
$$

Hence, we have for the repulsive solution

$$
\begin{gathered}
P_{1}=\frac{k^{(k+2) / k}}{k+2} \pi \frac{E^{*}}{b}\left(\frac{\delta_{1}^{k+2}}{R^{2(1-k)}}\right)^{1 / k} \\
A=k^{2 / k} \pi\left(\frac{\delta_{1}}{R^{1-k}}\right)^{2 / k}
\end{gathered}
$$

Computing derivatives $A, P_{1}^{\prime}, P_{1}^{\prime \prime}$ and using Eqs. (1) and (2) after some algebra the adhesive solution is obtained

$$
\begin{gathered}
P=\pi \frac{E^{*}}{b} \frac{k^{(k+2) / k}}{k+2}\left(\frac{\delta_{1}}{R^{1-k}}\right)^{2 / k}\left(\delta_{1}-\frac{k+2}{k} \sqrt{2 b \frac{w}{E^{*}}}\right) \\
\delta=\delta_{1}-\sqrt{2 b \frac{w}{E^{*}}}
\end{gathered}
$$

Under force control the pull-off is found imposing $P^{\prime}=0$ which gives 


$$
\delta_{1, P O}=\frac{2}{k} \sqrt{2 b \frac{w}{E^{*}}} ; \quad a_{P O}=\left(2 R^{k-1} \sqrt{2 b \frac{w}{E^{*}}}\right)^{1 / k}
$$

Substituting the first equation from Eqs. (12) into Eq. (10), the pull-off force is obtained

$$
P_{P O}=-2^{2 / k} \frac{k}{k+2} \pi \frac{E^{*}}{b} R^{2(k-1) / k}\left(2 b \frac{w}{E^{*}}\right)^{(2+k) /(2 k)}
$$

For a Hertzian profile, i.e. imposing $k=2$, the following results are obtained

$$
\delta_{1, P O}=\sqrt{2 b \frac{w}{E^{*}}} ; \quad P_{P O}=-2 \pi R w ; \quad a_{P O}=\sqrt{2 R}\left(\frac{2 b w}{E^{*}}\right)^{1 / 4}
$$

Notice that for the Hertzian case, the pull-off does not depend on the elastic properties of the layer, but only on geometry " $R$ " and surface energy " $w$ " similarly to the classical JKR results valid for a sphere pressed against an halfspace, but with an enhanced adhesive strength (in the classical JKR theory the pull-off is equal to $-3 / 2 \pi R w$ ). We define the dimensionless quantities

$$
\bar{\delta}=\frac{\delta}{\sqrt{2 b \frac{w}{E^{*}}}} ; \bar{P}=\frac{P}{2 \pi R w}
$$

Dimensionless forms of the adhesive load, Eq. (10), and pull-off reads

$$
\begin{gathered}
\bar{P}=\frac{k^{(k+2) / k}}{k+2} R^{(k-2) / k}\left(2 b \frac{w}{E^{*}}\right)^{(2-k) /(2 k)}(\bar{\delta}+1)^{2 / k}\left(\bar{\delta}-\frac{2}{k}\right) \\
\bar{P}_{P O}=-\frac{k}{k+2} 2^{2 / k} R^{(k-2) / k}\left(2 b \frac{w}{E^{*}}\right)^{(2-k) /(2 k)}
\end{gathered}
$$

where Eq. (11) is used to express Eq. (10) in terms of $\bar{\delta}$. For a given geometry of the indenter and for given material properties one may think to optimize the thickness of layer $b$. Notice that: for $k<2$ increasing the layer thickness leads to higher pull-off, for the Hertzian case $k=2$ the pull-off is insensitive to the layer thickness, while for $k>2$ the layer thickness has to be reduced for increasing the pull-off force. The layer thickness in fact is raised at the power $(2-k) /(2 k)$, which is plotted in Fig. 2 . For high values of exponent $k$ we obtain $\bar{P}_{P O} \propto b^{-1 / 2}$, which suggests that in order to obtain high adhesive strength $k>>2$ should be adopted together with a very thin layer. Bearing in mind that our argumentation is based on the thin layer approximation, in the rest of the paper we will focus on the case $k>2$. 


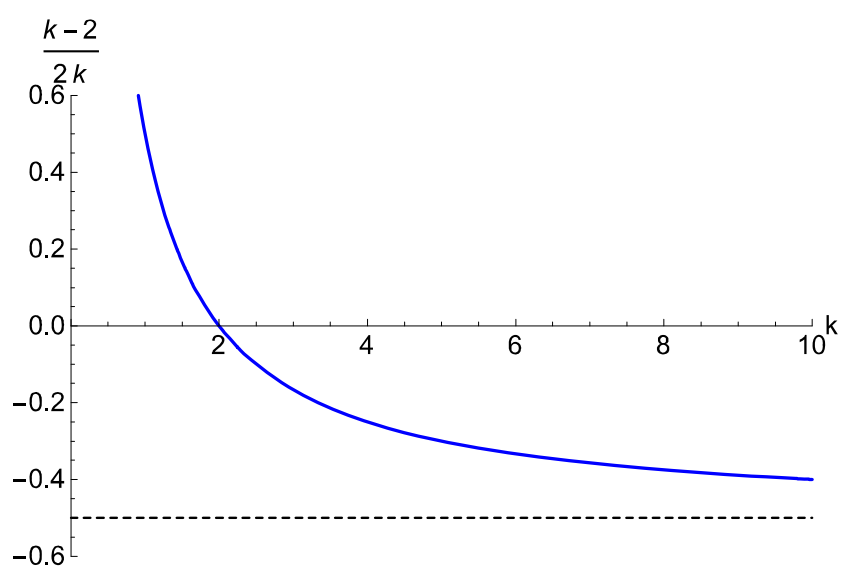

Fig. 2 The pull-off force is proportional to $\bar{P}_{P O} \propto b^{\frac{2-k}{2 k}}$. Here we plot the exponent $(2-k) /(2 k)$ versus $k$ to show that for $k<2(>2)$ thicker (thinner) layer increase the pull-off force

Similarly to Gao and Yao [7] we assume $w=10 \mathrm{~mJ} / \mathrm{m}^{2}, E^{*}=1 \mathrm{GPa}$ and for the layer thickness and reference length $b=1 \mu \mathrm{m}, R=1 \mathrm{~mm}$. The loading curves are reported in Fig. 3 for $k=[1.9,2,2.1]$. Two main points arise, i.e., firstly, there is no instability in displacement control (contrary to the classical JKR problem with halfspace), and secondly, the pull-off force is greatly affected by $k$. This is further confirmed in Fig. 4 where the pull-off force is plotted as a function of $k$ : it appears that moving from $k=2$ to $k=3$ an enhancement of one order of magnitude is obtained.

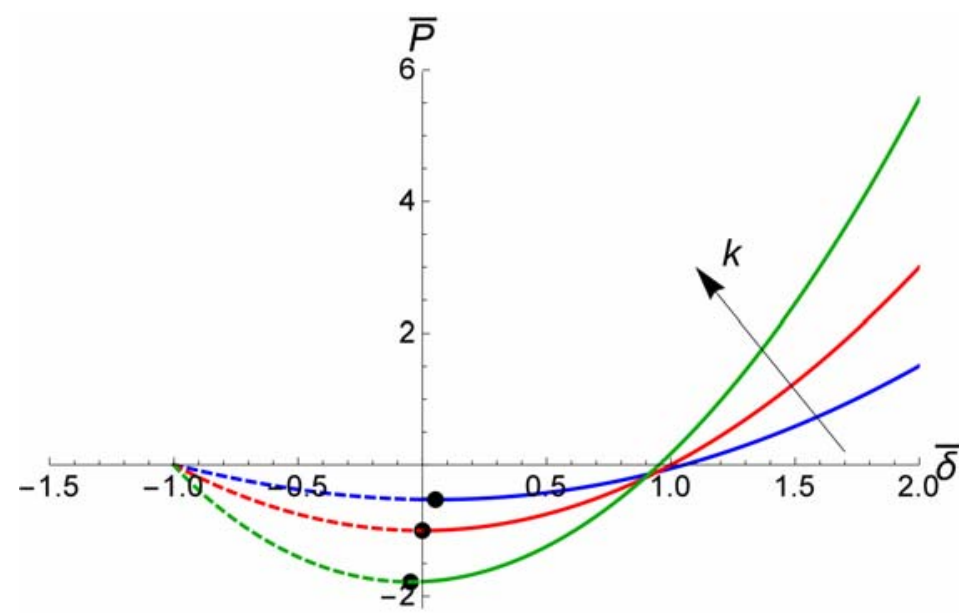

Fig. 3 Force-indentation curves for different power law profiles $k=[1.9,2,2.1]$. Solid (dashed) curves are stable (unstable) under force control 


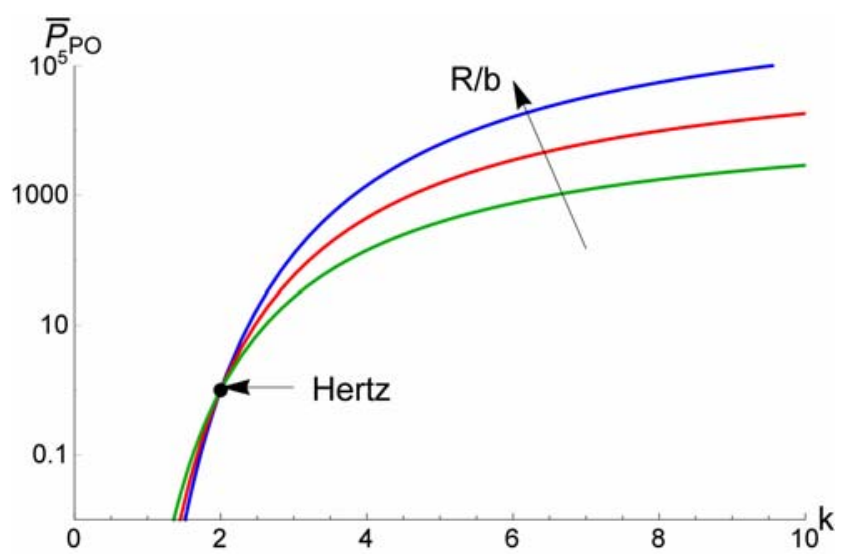

Fig. 4 Pull-off as a function of the indenter shape, $R / b=\left[10^{2}, 10^{3}, 10^{4}\right]$

The strong enhancement obtained increasing $k$ calls for further investigations. First we compute the average tension acting within the contact area at pull-off $\left|\sigma_{P O}\right|$

$$
\left|\sigma_{P O}\right|=\frac{\left|P_{P O}\right|}{\pi a_{P O}^{2}}=\frac{k}{k+2} \sqrt{\frac{2 E^{*} w}{b}}
$$

where we clearly recognize the toughness of material $K_{I c}=\left(E^{*} w\right)^{1 / 2}$ and the dependences on layer thickness $b^{-1 / 2}$ and the shape of indenter " $k$ ", but independent of the other lengthscale " $R$ " involved in the problem. For $k>2$, the "optimal" layer thickness, critical thickness $b_{c r}$ below which theoretical strength $\sigma_{t h}$ is reached, is easily obtained as

$$
b_{c r}=2\left(\frac{k}{k+2}\right)^{2}\left(\frac{K_{I c}}{\sigma_{t h}}\right)^{2}<<a_{P O}
$$

We shall assume reasonable values for $\sigma_{t h}=10 \mathrm{MPa}, w=10 \mathrm{~mJ} / \mathrm{m}^{2}, E^{*}=1 \mathrm{GPa}$ as in Gao and Yao [7] which for $k=2$ gives $b_{c r}=1 \mu \mathrm{m}$ and is further reduced for $k>2$. We recall here that the present analysis is valid within the Johnson's approximation $b<<a$. With the same set of parameters we estimate ratio $b / a_{\mathrm{PO}}$ in the "critical condition", i.e. for $b=b_{c r}=1 \mu \mathrm{m}$,

$$
\frac{b_{c r}}{a_{P O, c r}}=2^{1-2 / k}\left(\frac{k}{k+2}\right)^{2-1 / k} \frac{E^{*}}{\sigma_{t h}}\left(\frac{w / \sigma_{t h}}{R}\right)^{(k-1) / k}<<1
$$

and plot Eq. (20) in Fig. 5 as a function of $k$ and for $R / b=\left[10^{2}, 10^{3}, 10^{4}\right]$. The curves show that for $k>2$ the "optimal design" is feasible within the thin layer approximation where the pull-off tension is close to the theoretical strength of the material. Also notice that we have used $b=b_{c r}=1 \mu \mathrm{m}$, but for any $b<b_{c r} \rightarrow\left|\sigma_{\mathrm{PO}}\right|=\sigma_{\text {th; }}$; thus, the condition in Eq. (20) can be easily fulfilled for our reasonable set of parameter values. The "efficiency" of the adhesive mechanism is easily shown comparing the contact radius at pull-off with that obtained in the classical JKR theory, i.e. $a_{J K R, P O}=\left(9 / 8 \pi R^{2} w / E^{*}\right)^{1 / 3}$. In fact, ratio $a_{P O} / a_{J K R, P O} \propto b^{1 /(2 k)}$ and hence, for a sufficiently thin layer, the same detachment force is reached with a much smaller contact area. 


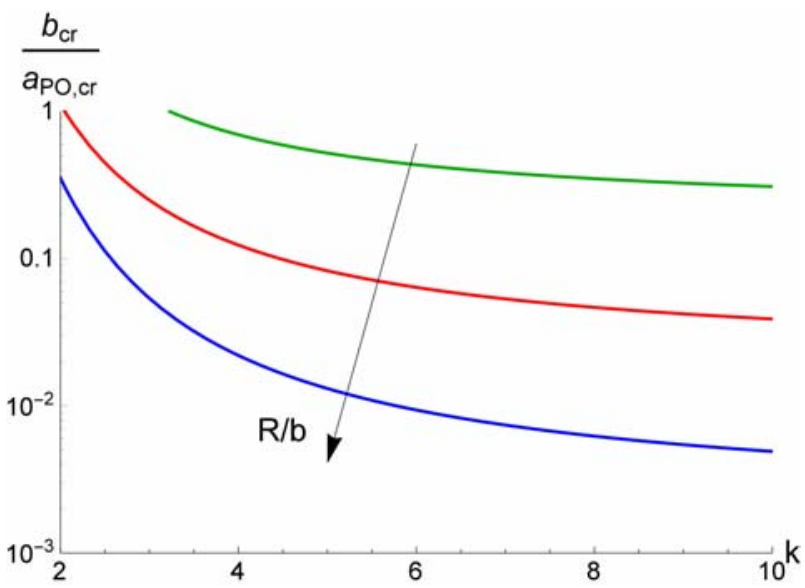

Fig. 5 Ratio $b_{c r} / a_{P O, c r}$ plotted for different geometries of the indenter, with $R / b=\left[10^{2}, 10^{3}, 10^{4}\right]$. The thin layer approximation is fulfilled for $b_{c r} / a_{P O, c r}<<1$

\subsection{Bonded compressible layer}

Barber [21] has shown that if the layer is compressible $v \neq 0.5$ and bounded to the rigid substrate, the contact pressure is given by Eq. (4), which differs from the frictionless behavior for a factor $Z=(1-v)^{2} /(1-2 v)$. Thus the previous analysis can be repeated to obtain the total adhesiveless load

$$
P_{1}=\frac{k^{(k+2) / k}}{k+2} \pi Z \frac{E^{*}}{b}\left(\frac{\delta_{1}^{k+2}}{R^{2(1-k)}}\right)^{1 / k}
$$

Computing derivatives $A, P_{1}{ }^{\prime}, P_{1}{ }^{\prime \prime}$ and using Eqs. (1) and (2) after some algebra the adhesive solution is obtained

$$
\begin{gathered}
P=\pi \frac{E^{*}}{b} Z \frac{k^{(k+2) / k}}{k+2}\left(\frac{\delta_{1}}{R^{1-k}}\right)^{2 / k}\left(\delta_{1}-\frac{k+2}{k} \sqrt{\frac{2 b}{Z} \frac{w}{E^{*}}}\right) \\
\delta=\delta_{1}-\sqrt{\frac{2 b}{Z} \frac{w}{E^{*}}}
\end{gathered}
$$

which corresponds to the Hertzian case for $k=2$. To find the minimum we impose $P^{\prime}=0$, which gives

$$
\delta_{1, P O}=\frac{2}{k} \sqrt{\frac{2 b}{Z} \frac{w}{E^{*}}} ; \quad a_{P O}=\left(2 R^{k-1} \sqrt{\frac{2 b}{Z} \frac{w}{E^{*}}}\right)^{1 / k}
$$

and hence

$$
P_{P O}=-2^{2 / k} \frac{k}{k+2} \pi \frac{E^{*}}{b} R^{2(k-1) / k} Z^{(k-2) /(2 k)}\left(2 b \frac{w}{E^{*}}\right)^{(2+k) /(2 k)}
$$


Notice that for $k>2$ pull-off $P_{P O}$ is increased and contact area $\pi a_{P O}^{2}$ decreased with respect to the frictionless case, thus, due to Poisson's effects, the contact is more "efficient" in the bounded configuration.

\section{CONCLUSIONS}

In this paper we have studied the adhesive indentation by an axisymmetric frictionless rigid indenter with power-law profile on a thin layer supported by a rigid foundation. It has been shown that the detachment force at pull-off is strongly affected by the geometry of the tip. Nevertheless we noted that reducing the layer thickness, which also fulfills the "thin layer approximation", leads to reaching the theoretical strength at pull-off. Using reasonable data for the elastic and adhesive parameters of the layer, as in Gao and Yao [7], we have shown that the critical thickness of the layer below which the theoretical strength is reached is of the order of $1 \mu \mathrm{m}$. While for the case of Hertzian (parabolic) profile the detachment force is independent of the thickness of the layer and on its elastic properties (similarly to the classical results of Johnson-Kendal-Roberts valid for halfspace geometry) when a general power-law is used, this dependence arises. It has been shown that the adhesive mechanism is more efficient when compared to the JKR halfspace solution, particularly for bounded compressible layers, as the same detachment force is obtained with a much smaller contact area. This occurs because the dominant length scale for the stress intensity factor at the contact edge is the layer thickness. The presented analysis is particularly suited for polymeric coating of metallic samples with micro or nanometer thickness. Exploiting different probe profiles high sensitivity micro-/nanoindentation test may be performed to determine adhesive and elastic properties $\left(w, E^{*}\right)$ of the thin layers coated on "rigid" substrates. Possible extension of the present work may consider the adhesive indentation of multilayered systems, which are of interest in tribology, as for the "Surface Force Apparatus", often represented as a three-layer halfspace [27].

\section{REFERENCES}

1. Pastewka, L., Robbins, M.O., 2014, Contact between rough surfaces and a criterion for macroscopic adhesion. Proceedings of the National Academy of Sciences, 111(9), pp. 3298-3303.

2. Ciavarella, M., Papangelo, A., 2018, A generalized Johnson parameter for pull-off decay in the adhesion of rough surfaces, Physical Mesomechanics, 21(1), pp. 67-75.

3. Ciavarella, M., Papangelo, A., 2018, A modified form of Pastewka--Robbins criterion for adhesion, The Journal of Adhesion, 94(2), pp. 155-165.

4. Ciavarella, M., Papangelo, A, 2017, A random process asperity model for adhesion between rough surfaces, Journal of Adhesion Science and Technology, 31(22), pp. 2445-2467.

5. Huber, G, Gorb, S, Hosoda, N, Spolenak, R, Arzt, E., 2007, Influence of surface roughness on gecko adhesion, Acta Biomater., 3, pp. 607-610.

6. Pugno, N.M., Lepore, E., 2008, Observation of optimal gecko's adhesion on nanorough surfaces. Biosystems, 94, pp. 218-222.

7. Gao, H., Yao, H., 2004, Shape insensitive optimal adhesion of nanoscale fibrillar structures, Proceedings of the National Academy of Sciences of the United States of America, 101(21), pp. 7851-7856.

8. Yao, H., Gao, H., 2006, Optimal shapes for adhesive binding between two elastic bodies, Journal of colloid and interface science, 298(2), pp. 564-572.

9. Papangelo, A., Afferrante, L., Ciavarella, M., 2017, A note on the pull-off force for a pattern of contacts distributed over a halfspace, Meccanica, 52(11-12), pp. 2865-2871. 
10. Akerboom, S., Appel, J., Labonte, D., Federle, W., Sprakel, J., Kamperman, M.., 2015, Enhanced adhesion of bioinspired nanopatterned elastomers via colloidal surface assembly, Journal of The Royal Society Interface, 12(102), doi:10.1098/rsif.2014.1061.

11. Papangelo, A., Ciavarella, M., 2017, A Maugis-Dugdale cohesive solution for adhesion of a surface with a dimple, Journal of The Royal Society Interface, 14(127), doi: 10.1098/rsif.2016.0996.

12. Papangelo, A., Ciavarella, M., 2018, Adhesion of surfaces with wavy roughness and a shallow depression, Mechanics of Materials, 118, pp. 11-16.

13. Yang, F., 2002, Adhesive contact between a rigid axisymmetric indenter and an incompressible elastic thin film, Journal of Physics D: Applied Physics, 35(20), pp. 2614-2620.

14. Yang, F., 2006, Asymptotic solution to axisymmetric indentation of a compressible elastic thin film, Thin Solid Films, 515(4), pp. 2274-2283.

15. Reedy, E.D., 2006, Thin-coating contact mechanics with adhesion, Journal of Materials Research, 21(10), pp. 2660-2668.

16. Johnson, K.L., Kendall, K., Roberts. A.D., 1971, Surface energy and the contact of elastic solids, Proc Royal Soc London A, 324(1558), doi: 10.1098/rspa.1971.0141.

17. Popov, V.L., Heß, M., 2015, Method of dimensionality reduction in contact mechanics and friction, Springer, Berlin Heidelberg.

18. Argatov, I.I., Mishuris, G.S., Popov, V.L., 2016, Asymptotic modelling of the JKR adhesion contact for a thin elastic layer, The Quarterly Journal of Mechanics and Applied Mathematics, 69(2), pp. 161-179.

19. Johnson, K.L., 1985, Contact Mechanics, Cambridge University Press, Cambridge.

20. Jaffar, M. J., 1989, Asymptotic behaviour of thin elastic layers bonded and unbonded to a rigid foundation, Int. J.Mech. Sci. 31(3), pp. 229-235.

21. Barber, J.R., 1990, Contact problems for the thin elastic layer, International Journal of Mechanical Sciences, 32(2), pp. 129-132.

22. Argatov, I., Li, Q., Pohrt, R., Popov, V.L., 2016, Johnson-Kendall-Roberts adhesive contact for a toroidal indenter, Proc. R. Soc. A 472: 20160218.

23. Willert, E., Li, Q., Popov, V.L., 2016, The JKR-adhesive normal contact problem of axisymmetric rigid punches with a flat annular shape or concave profiles, Facta Universitatis-Series Mechanical Engineering, 14(3), pp. 281-292.

24. Popov, V.L., Heß, M., Willert, E., 2017, Handbuch der Kontaktmechanik: Exakte Lösungen axialsymmetrischer Kontaktprobleme, Springer, Berlin, 341 p.

25. Ciavarella, M., 2017, An approximate JKR solution for a general contact, including rough contacts, arXiv preprint arXiv:1712.05844

26. Popov, V.L., Pohrt, R., Li, Q., 2017, Strength of adhesive contacts: Influence of contact geometry and material gradients, Friction, 5(3), pp. 308-325.

27. Sridhar, I., Johnson, K.L., Fleck, N.A., 1997, Adhesion mechanics of the surface force apparatus, Journal of Physics D: Applied Physics, 30(12), pp. 1710-1719. 